Check for updates

Cite this: RSC Adv., 2017, 7, 56108

Received 15th October 2017

Accepted 6th December 2017

DOI: $10.1039 / c 7 r a 11357 a$

rsc.li/rsc-advances

\section{Functional human 3D microvascular networks on a chip to study the procoagulant effects of ambient fine particulate matter}

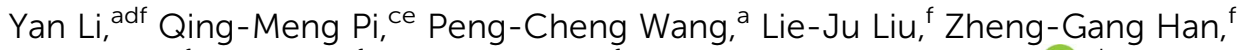 \\ Yang Shao, ${ }^{f}$ Ying Zhai, ${ }^{f}$ Zheng-Yu Zuo, ${ }^{f}$ Zhi-Yong Gong, ${ }^{a}$ Xu Yang (D) *b \\ and Yang Wu (D) *a
}

Exposure to ambient fine particulate matter (FPM) has been thought to be associated with cardiovascular disease. However, the pathogenesis remains largely unknown. Animal models have been widely used in toxicological research, but species difference makes it impossible to directly translate discoveries from animals to humans. In this study, we developed a 3D functional human microvascular network in a microfluidic device. The established model enables endothelial cells to form vessel-like microtissues and have physiological functions which are closer to cells in human blood vessels. The perfusable microvasculature allows the delivery of nutrients, and oxygen, as well as flow-induced mechanical stimuli into the luminal space of the endothelium. The microflow effectively mimic the blood flow in human vessels. FPMs were introduced into this physiologically human vessel-like microenvironment following the fluid flow. The vascular toxicity was evaluated based on this organotypic 3D microvessel model. Our results demonstrated that intravascular accumulation of FPM could enhance ROS generation which may further cause endothelial dysfunction by oxidative stress. This is expressed in disorder of NO expression and IL-6 up-regulation. These are expected to enhance endothelial inflammation which might in turn accelerate coagulation that is associated with thrombosis. Human organotypic 3D microvessel models provide a possible bridge for how the research outcomes translate to humans. These models could partly simulate the physiological responses of human vessels to FPM stimulation. This simple and versatile platform can be used for a wide range of applications in vascular physiology studies of particulate matter in the context of cardiovascular disease.

\section{Introduction}

Air pollution is an increasing risk for cardiovascular morbidity and mortality in humans., ${ }^{1,2}$ Numerous epidemiological and clinical studies indicate that ambient particulate matter (PM) in air pollution is strongly associated with increased

\footnotetext{
${ }^{a}$ Key Laboratory for Deep Processing of Major Grain and Oil (Wuhan Polytechnic University), Ministry of Education, College of Food Science and Engineering, Wuhan Polytechnic University, Wuhan 430023, PR China. E-mail: wuyangwhpu@163.com; Fax: +86-27-83956793 ext. 208; Tel: +86-13871548015

${ }^{b}$ Hubei Key Laboratory of Genetic Regulation and Integrative Biology, College of Life Sciences, Central China Normal University, Wuhan 430079, PR China. E-mail: yanxgu@mail.ccnu.edu.cn; Fax: +86-27-67861936; Tel: +86-13871361954

'Harvard-MIT Division of Health Sciences and Technology, Massachusetts Institute of Technology, Cambridge, MA 02139, USA

${ }^{d}$ Department of Biomedical Engineering, School of Engineering \& Applied Science, Yale University, New Haven 06520, USA

${ }^{e}$ Department of Orthopedic Surgery, Shanghai Jiaotong University Affiliated Sixth People's Hospital, Shanghai Jiaotong University, Shanghai 200233, PR China

${ }^{f}$ School of Biology and Pharmaceutical Engineering, Wuhan Polytechnic University, Wuhan 430023, PR China
}

cardiovascular disease. ${ }^{3,4}$ And PM induced coagulation abnormalities are very likely responsible for many kinds of cardiovascular disease., ${ }^{5,6}$ The precise pathway explaining how PM disrupts the coagulation function has, however, not yet been entirely determined. Recent studies have increasingly focused on ambient fine particulate matter (FPM). Nanoscale fine particles facilitate their migration across any biological barrier and thus enter organs. ${ }^{7,8}$ Once inhaled, FPM can more easily cross the pulmonary epithelium and the lung-blood barrier than the micron sized-PM. ${ }^{9}$ Therefore, FPM is considered to be a major contributor in the disruption of human vascular function.

The translocation of FPM to the blood stream has detrimental effects on the cardiovascular system. Previous studies have revealed the potential relationships between FPM and cardiovascular disease. Once FPM has translocated into the blood stream, this FPM is expected to exhibit particulate physiological characteristics due to their tiny size and special structure. ${ }^{10}$ FPM has a relatively large surface area, and may therefore exhibit extensive surface activity and absorb protein rich fluids in vivo. ${ }^{11}$ This kind of biointeraction may affect 
protein function ${ }^{12}$ and cytokine expression, ${ }^{13}$ which potentially may further disturb normal vascular function. In addition, research has also suggested that FPM can aggravate local oxidative stress and inflammation after FPM is deposited on the vascular endothelium. ${ }^{14}$ Based on an animal model, FPM may induce aberrant expression of reactive oxygen species (ROS),$^{15}$ endogenous nitric oxide (NO) ${ }^{16}$ and interleukin 6 (IL-6). ${ }^{17}$ This bioresponse could further result in plaque activation, and finally lead to thrombus formation. ${ }^{18}$

Animal models have been widely used in toxicological research, and they continue to aid our understanding of PM associated cardiovascular disease. ${ }^{19}$ However, we need to recognize that there are potential differences between an animal model and humans. Even animals that are very similar to humans, such as primates, have failed to predict what will happen in humans. ${ }^{20}$ Therefore, it is impossible to directly translate discoveries from animals to humans. Human organotypic 3D cell cultures provide an attractive alternative to animal models from an applicability perspective. ${ }^{21,22}$ As compared to endothelial cells cultured on a flat surface (2D culture), 3D cultures provide a more physiologically tissue-like environment for endothelial cells. ${ }^{23}$ The $3 \mathrm{D}$ cultures allow human endothelial cells in vitro to generate microvascular networks which are similar to human vessels. ${ }^{24}$ This organotypic cell model can more faithfully provide comprehensive and relevant biological information than can be obtained from 2D models.

In recent years, a scaffold-based 3D cell culture has been generally adopted by researchers. Scaffolds mediate the adhesion, proliferation and differentiation of cells. ${ }^{25}$ Scaffolds also represent the 3D space which is available for cells developing into tissues, and for defining the shape and function of regenerative tissue. ${ }^{26}$ Fibrin based scaffolds are approved to be biocompatible, while degrading and resorbing at a rate corresponding to tissue growth. ${ }^{27}$ The combination of fibrin hydrogel with a microfluidic device has been widely investigated for the regeneration of human vessel tissue. ${ }^{28}$ The microfluidic chip can provide micro-scale complex structures and well-controlled parameters to mimic the in vivo environment of human vessels. For example, microfluid flow is able to supply the necessary nutrition, moisture and oxygen, as well as being able to remove degradation products at the same time. ${ }^{29,30}$ Microfluid flow was shown to generate improvement in the adhesion, migration and proliferation of human endothelial cells in 3D scaffolds. ${ }^{31,32}$ Accordingly, the perfusable microvascular networks were spontaneously formed in this 3D microenvironment.

The generation of functional microvascular networks is critical for in vitro models to replicate pathophysiological conditions. In this study, perfusable microvascular networks were made by co-culturing human umbilical vein endothelial cells (HUVECs) and human lung fibroblasts (HLFs) with 3D fibrin hydrogels which were loaded in the microfluidic devices. This device offers a physiologically tissue-like in vitro platform to investigate any human vascular dysfunction resulting from FPM stimulation. The morphology, components and size of the FPM were analyzed. Taking into account established exposure level, we used FPM concentrations of 5, 10 and $20 \mu \mathrm{g} \mathrm{ml} \mathrm{m}^{-1}$ for the functional microvascular networks. We observed and analyzed the procoagulant effects on the microvascular networks after FPM stimulation. These responses include reactive oxygen species (ROS), endothelial nitric oxide (NO) and interleukin 6 (IL-6). The objective was to gain a better insight and understanding of FPM associated human vascular dysfunction.

\section{Results and discussion}

\subsection{Physicochemical characteristics of FPM}

The inhalation of air containing fine particulate matter is associated with cardiovascular disease in humans. The small particle size facilitates the passage of FPM through any biological barrier, and enables these particles to enter the hematological system. Atomic force microscopy (AFM) can identify nanoscale particles in air pollution samples. ${ }^{33}$ In this research, the initial air samples were characterized using an atomic force microscope. Of all the observed particles, AFM demonstrated that all of the collected particles had a nanoscale aerodynamic diameter. The AFM image clearly showed that most of the particles are in the tens to hundreds nanometer range (Fig. 1A). The three-dimensional morphology of the airborne nanoscale particles was also characterized by AFM. The morphology of
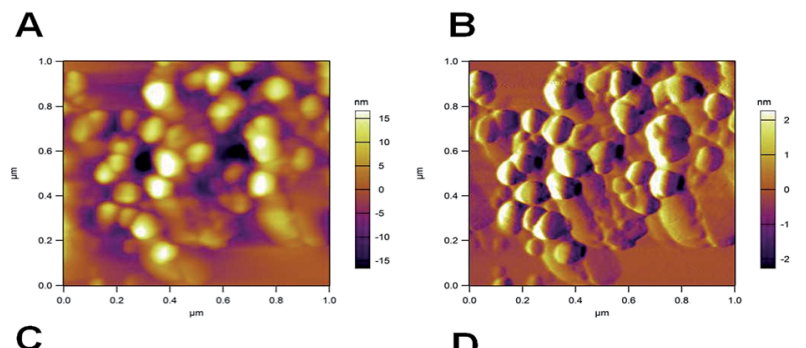

C
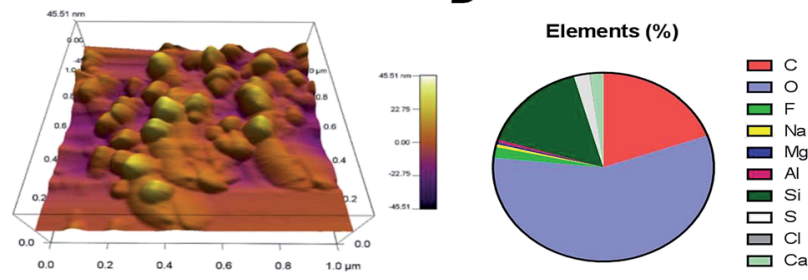

E

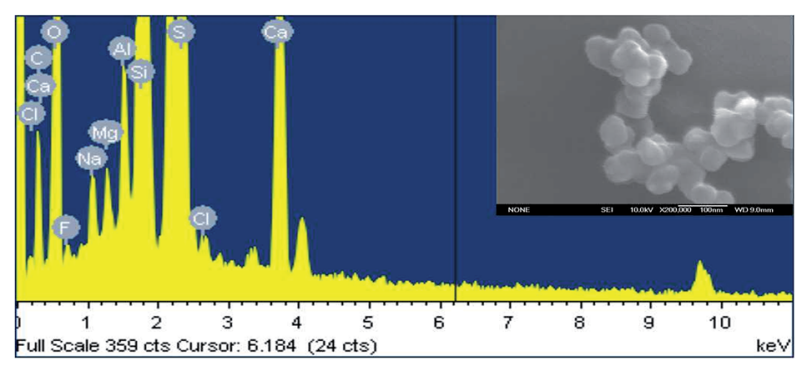

Fig. 1 Physicochemical characterization of ambient fine particulate matter (FPM). (A-C) Atomic force microscopy image of FPM; (D) chemical element analysis of FPM by energy dispersive $X$-ray analysis (EDAX); (E) SEM picture of ambient fine particulate matter and EDAX analysis of one representative particle. 
every single FPM particle is shown in Fig. 1B. Fig. 1C gives 3D version of airborne nanoscale particles. The distribution of the main chemical elements of the particles was analyzed using energy dispersive X-ray analysis (EDAX) spectrometry. Fig. 1E is SEM picture of ambient nanoscale particles and EDAX analysis of one representative particle. One hundred particles were analyzed by this method. EDAX analysis showed that our collected FPMs consisted mainly of oxygen (57.14\%), carbon (19.53\%) and silicon (15.9\%) (Fig. 1D). As compared to micron size particles, nanoscale particles are believed to exhibit unique physicochemical properties. Because nanoparticles have a high surface-specific activity which may lead to greater proinflammatory responses. ${ }^{34}$ Therefore, the existence of airborne FPM is recognized to be a potential risk to humans because of the ability of FPM to translocate from the deposition site to the blood system.

\subsection{Human vessel simulation by 3D organotypic HUVEC culture}

Human coagulation abnormalities are reported to be associated with FPM in blood vessels. ${ }^{35}$ However, animal models are poorly extrapolating the results to humans directly. ${ }^{36,37}$ Human organotypic $3 \mathrm{D}$ cell culture is an attractive alternative to traditional animal models. It provides a bridge for how research outcomes may translate to humans. In this research, we compared a $2 \mathrm{D}$ cell culture with a $3 \mathrm{D}$ cell culture. In the $2 \mathrm{D}$ cell culture, HUVECs were grown on flat dishes. The cells were adhered and spread on this plastic surface and formed unnatural cell attachments to the synthetic surface (Fig. 2A and B). The essential cellular functions that are present in tissues are not found in the presence of this kind of unnatural cell adherent. ${ }^{38}$
A

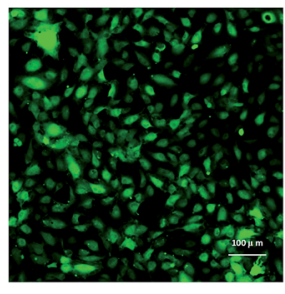

C

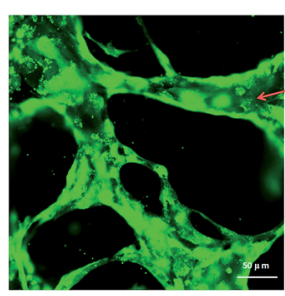

B
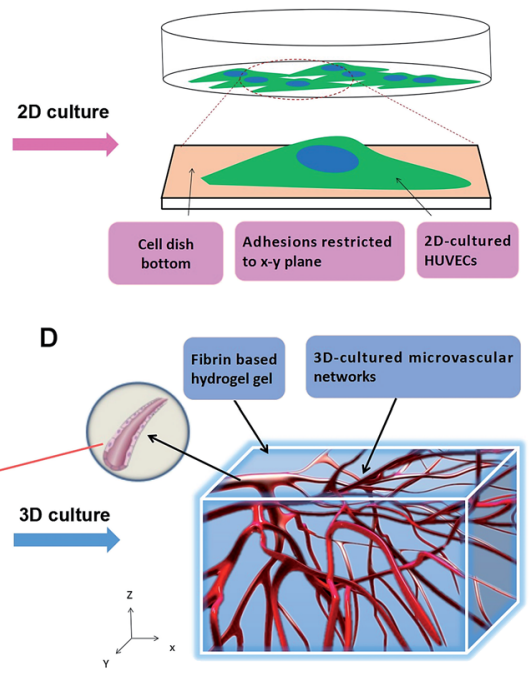

Fig. 2 2D versus 3D cultures of human umbilical vein endothelial cells (HUVECS). (A) Confocal microscope image of 2D cultured HUVECs; (B) schematic diagram of 2D culture: HUVECs attached to cell dish bottom; (C) confocal microscope image of 3D microvascular network; (D) schematic diagram of 3D HUVECs culture: microvessels generate in fibrin based scaffold.
In contrast, HUVEC cultured in a $3 \mathrm{D}$ model maintains a physical structure, ${ }^{39}$ and can closely mimic the functions of living tissues present in the human body. ${ }^{40}$ As shown in Fig. $2 \mathrm{C}$ and D, HUVECs grown in vitro formed capillary-like structures with lumens. The microvascular tissue was regenerated in vitro based on 3D organotypic HUVEC culture.

It provides a more biologically faithful platform in vitro as compared to traditional 2D cell modeling. Meanwhile, it also regenerated a high homologous human vessel-like microtissue in vitro. In this research, we used fibrin gel to generate scaffolds for the 3D organotypic culture of endothelial cells. The advantages of fibrin gel over synthetic polymeric materials are that fibrin gel is nontoxic, and has controllable degradation and excellent biocompatibility. ${ }^{41}$

\subsection{Microvascular network formation based on microfluidic 3D HUVEC culture}

We have used a combination of fibrin hydrogel with a microfluidic device in this research to promote microvascular formation. Cells were mixed with a fibrin precursor and thrombin. The mixtures were delivered into a gel filling channel and cultured for 40 minutes to complete the gelation (Fig. 3A). After gelation, $70 \mu \mathrm{l}$ of the HUVEC medium was introduced into
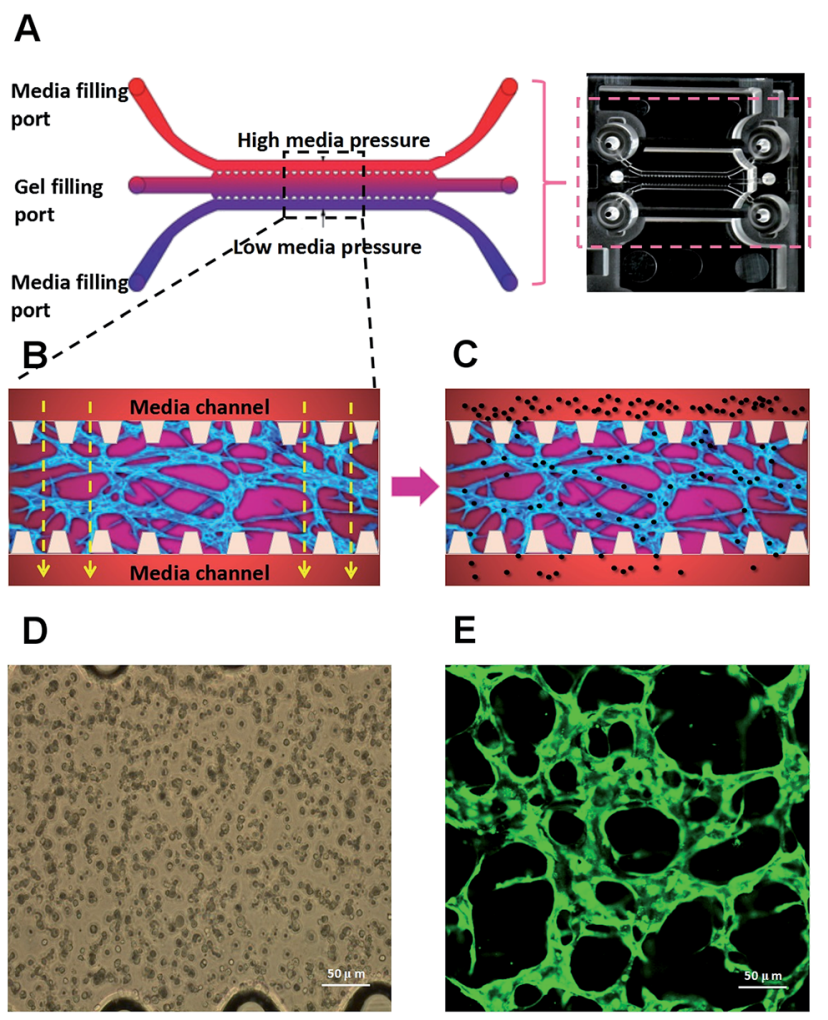

Fig. 3 Microvascular network formation based on microfluidic 3D HUVEC culture. (A) Schematic diagram of a microfluidic device. (B) Schematic diagram of microvascular network formation based on microfluidic 3D HUVEC culture. (C) Schematic diagram of loading microparticles in microvascular networks. (D) Microscope image of HUVECs seeding in fibrin hydrogel. (E) Confocal microscope image of fluorescent microvascular networks. 
any two reservoirs connected to the same media channel. $60 \mu \mathrm{l}$ of the medium was introduced into the other two reservoirs later. A difference in liquid levels in the media channels creates a pressure drop along the gel filling channel (Fig. 3B). The liquid pressure enables the EGM-2 medium to permeate into the fibrin hydrogel. With the aid of fluid flow, the microfluidic device offers a dynamic microenvironment for 3D HUVE culture. Besides that, the fluid shear stresses are already approved to promote endothelial proliferation and microvessels generation. The human vascular system enables gas exchange in the body ${ }^{\mathbf{4 2}}$ as well as the transport of nutrients, waste, pathogens and blood cells. ${ }^{43}$ In this microfluidic system, fluid flow ensures continuous nutrition, oxygen supply, and waste removal through the medium pressure of media channels. Microfluid flow has been shown to generate improvement in the adhesion, migration and proliferation of HUVECs in fibrin hydrogel. As shown in Fig. 3D and E, HUVECs seeded in fibrin hydrogel (Fig. 3D) spontaneously formed microvascular networks (Fig. 3E) in the microfluidic device. In addition, fluid flow is also able to import FPM in the 3D microvascular network (Fig. 3C), which effectively simulates the bioprogress of the FPM translocating into a human blood vessel.

In this research, HUVECs and HLFs were co-cultured with fibrin hydrogel in a microfluidic device. Fibroblasts are proven to secrete growth factors that support endothelial cell form lumen in three-dimensional co-cultures. ${ }^{\mathbf{4 4 , 4 5}}$ In the presence of fibroblasts, HUVEC spreading is robust and forms intracellular lumen within four to five days. As shown in Fig. 4, 3D-cultured HUVEC development was monitored using a camera in
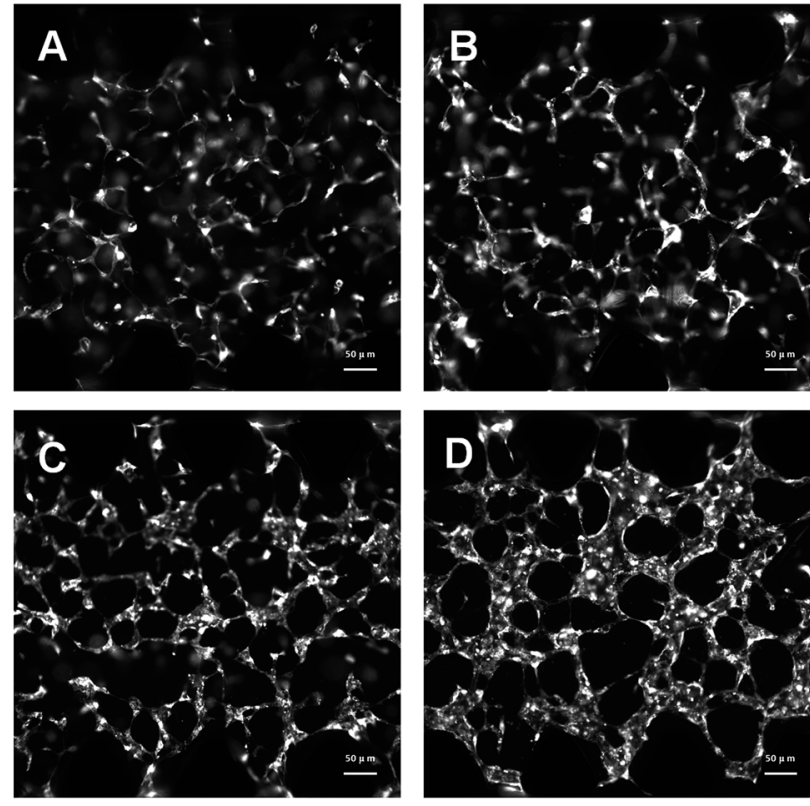

Fig. 4 Development progress of microvascular networks were monitored by a camera in a confocal microscope. (A) Camera image of fluorescent HUVECs developed on day 1; (B) camera image of fluorescent HUVECs developed on day 2; (C) camera image of fluorescent HUVECs developed on day 3; (D) camera image of fluorescent HUVECs developed on day 4 a confocal microscope. In the initial stage, HUVECs migrated to form cell-cell adhesions and alignments (Fig. 4A and B). After that, HUVECs gradually formed lumens along with cell proliferation (Fig. 4C). Finally, HUVEC lumens formed microvascular networks (Fig. 4D). In contrast, some research has indicated that HUVECs do not form vessels if fibroblasts are not present, and most HUVECs would be dead after being cultured in hydrogel for four to five days. ${ }^{46}$

\subsection{Perfusion measurement of 3D human microvascular networks}

In a living body, perfusion refers to the flow of blood through the vessels of the body. It ensures the blood flows through the circulatory system or lymphatic system to an organ or tissue. ${ }^{47}$ Adequate perfusion of the microvasculature has important physiological significance. ${ }^{48}$ It ensures the transport of dissolved gases, nutrients and regulatory molecules throughout the human microvasculature as well as the prompt removal of metabolic waste products. ${ }^{49}$ Perfusion of $3 \mathrm{D}$ cultured microvascular networks is almost exclusively reserved for microfluidic techniques. ${ }^{50}$ In this research, apart from replicating vessel-like structure, the perfusion capability has been measured for the microvascular networks in microfluidic device. Fluorescent microparticles (FM) were initially loaded into any two reservoirs connected to the same media channel. The liquid pressure enabled the FM to permeate into the microvascular network (Fig. 5A). As shown in Fig. 5A, it is schematic diagram to show

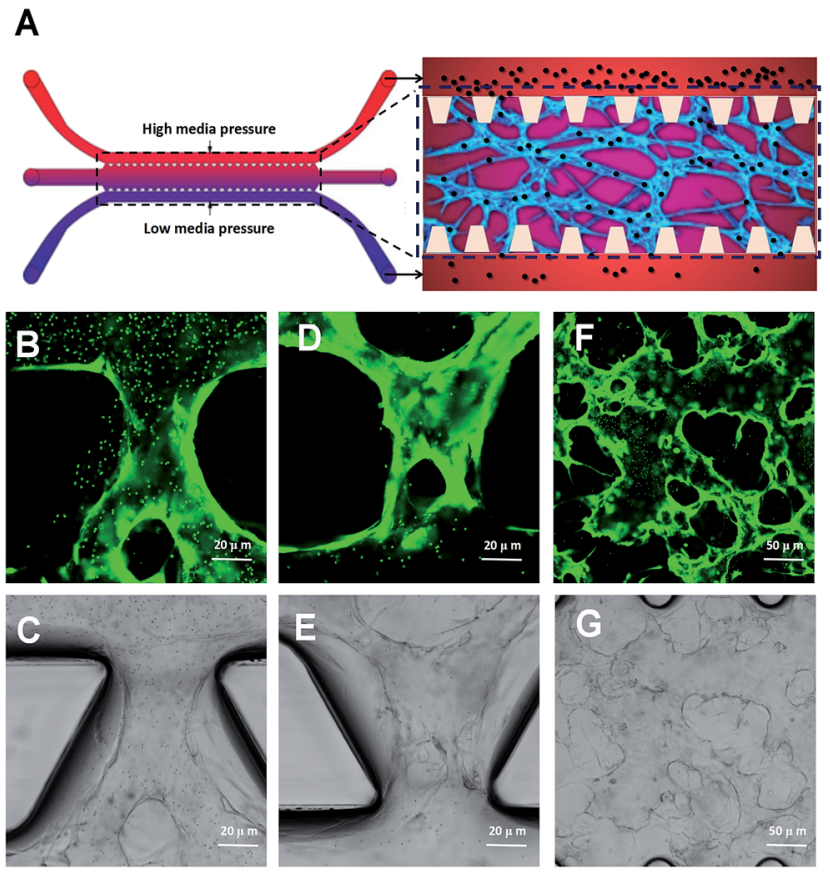

Fig. 5 Perfusion measurement of a 3D-cultured human microvascular network. (A) Schematic diagram of loading microparticles in microvascular networks. (B-C) Confocal images of fluorescent microparticles perfused in the microvascular network from the upstream direction. (D-E) Confocal images of fluorescent microparticles discharged downstream. (F-G) Confocal microscope image of fluorescent microparticles moving through the microvascular networks. 
how the loading microparticles move in microvascular networks and migrate through vessels following with fluid flow. Fig. 5A illustrates the running track of microparticles in microvessels. With the aid of fluid flow, the microfluidic device provides the dynamic power that enables the FM to go through the artificial human vessels. FMs perfused in the microvascular network from the upstream direction (Fig. 5B and C). FMs were discharged downstream along with fluid flow (Fig. 5D and E). Panorama images further confirmed that FMs were moving through the microvascular networks (Fig. 5F and G). By tracking FM movement, the microvascular network exhibited great perfusion ability. Perfusion is one of the crucial aspects in vasculature, as it provides shear stress, which affects cellular morphology and gene expression. ${ }^{51}$ Benefits associated with perfusion flow include a stable nutrient supply, waste metabolites removal and control of oxygen tension. In this research, the constant perfusion of media throughout the thick hydrogel significantly improved the viability of HUVECs forming capillary-like microvascular networks.

\subsection{A dynamic study of intravascular particle accumulation}

Previous evidences suggest that inhaled insoluble nanoparticles may be capable of translocation into the vascular system. ${ }^{52}$ Based on animal models, exogenous substance could be removed by the cells of the reticuloendothelial system/ mononuclear phagocyte system..$^{53,54}$ However, due to the primary size and degree of particle aggregation, nanoscale may lead to the FPM clearance more or less effective. In this research, we generated perfusable microvascular networks based on 3D HUVEC cultures in microfluidic systems. The
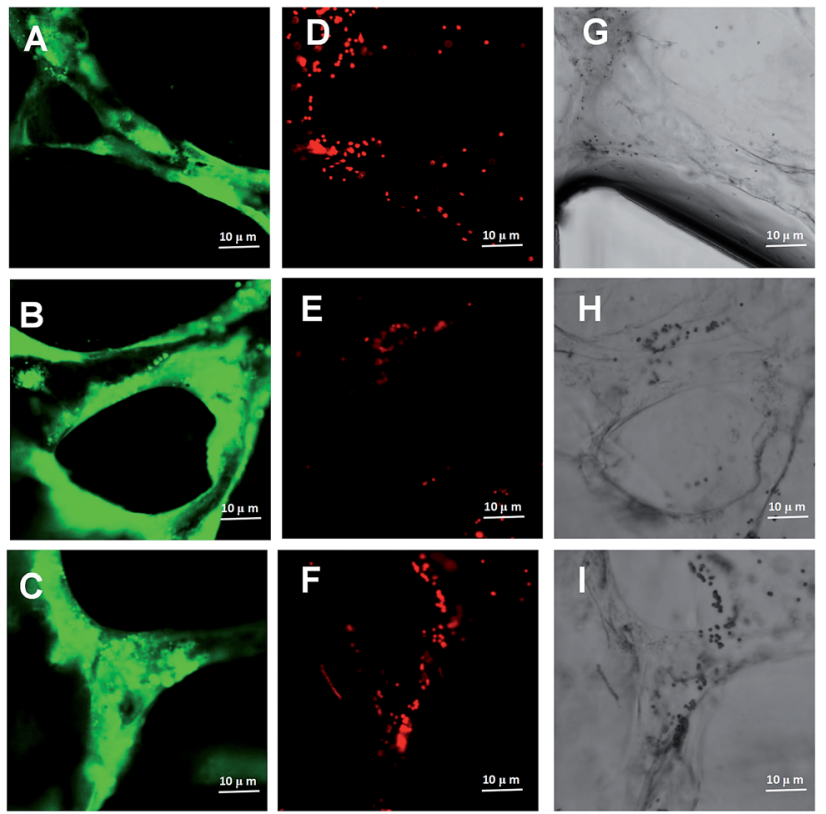

Fig. 6 A dynamic study of intravascular particle accumulation. (A-C) Confocal images of fluorescent microvascular networks. (D-F) Confocal images of fluorescent microparticle accumulation. (G-I) Combined confocal images of fluorescent microparticle accumulation within microvascular networks. microscale dimensions of the $3 \mathrm{D}$ vessels match the vascular structures inside the human body (Fig. 6A-C). The replicated human vessels can be created to mimic the complex and dynamic 3D network present in real human blood systems. We used fluorescent microparticles loaded into the microvascular networks. Following the fluid flow, this platform makes it possible to simulate the dynamics of FPM movement in a human blood vessel. As fluorescent microparticles perfuse into the microvascular network with the fluid flow, some microparticles aggregated and blocked local parts of the capillaries (Fig. 6D-F). The confocal microscope images clearly demonstrated an intravascular accumulation of fluorescent microparticles (Fig. 6G-I). This observed phenomenon revealed one possible pathological mechanism behind FPM's procoagulant ability. This ability is due to great surface energy, FPMs are constantly gathering and form local intravascular accumulation. ${ }^{55}$ It is potentially the result of a blood pressure increase due to the vessel lumen narrowing. As blood pressure increases, the shear forces within the vascular lumens of large vessels increase, creating more friction, which increases inflammation to these vessels. ${ }^{56}$ Inflammation plays an important role in the pathophysiology of thrombosis. Systemic inflammation is generally accepted as a potent prothrombotic stimulus. ${ }^{57}$ It upregulates the procoagulant factors, downregulates natural anticoagulants and inhibits fibrinolytic activity. ${ }^{58}$ In addition to modulating plasma coagulation mechanisms, inflammatory mediators also appear to increase platelet reactivity, which might somehow activate the coagulation. ${ }^{59}$

\subsection{Measurement of FPM induced endothelial dysfunction based on a 3D human microvascular model}

Previous inflammation-induced thrombosis is a well-known entity based on animal models. ${ }^{60}$ In the pathogenesis progress, inflammation and coagulation play a pivotal role. In this research, a dynamic study of microparticle movement has been conducted using 3D-human microvascular networks. Within the vascular compartment, particles encounter vessels, and this encounter is expected to induce oxidative stress in endothelial cells. ${ }^{61}$ The vascular endothelium, which regulates the passage of macromolecules and circulating cells from blood to tissues, is a major target of oxidative stress, and plays a critical role in the pathophysiology of vascular dysfunction. ${ }^{62}$ Based on the $3 \mathrm{D}$ human microvascular model, HUVECs were exposed to FPMs in a physiologically human blood vessel-like environment. The intravascular ROS increased in a dose dependent manner with FPM concentrations (Fig. 7A). Administration of an antioxidant (vitamin C) is seen to effectively repress ROS generation (Fig. 7B). ROS are key signaling molecules that play an important role in the progression of oxidative stress and inflammatory disorders. ${ }^{63}$ Our results demonstrated that intravascular accumulation of FPM could enhance ROS generation which may further cause endothelial dysfunction.

Endothelial dysfunction is commonly associated with decreased bioavailability of endothelial NO, which is a result of impaired NO production by the endothelium and/or increased inactivation of NO by ROS. ${ }^{64,65}$ In this research, endothelial NO 
A

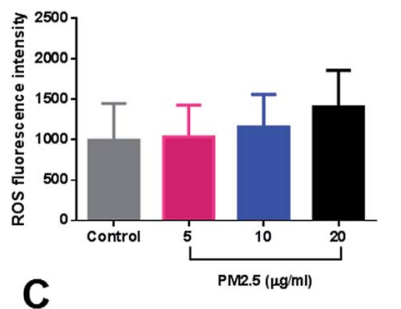

C
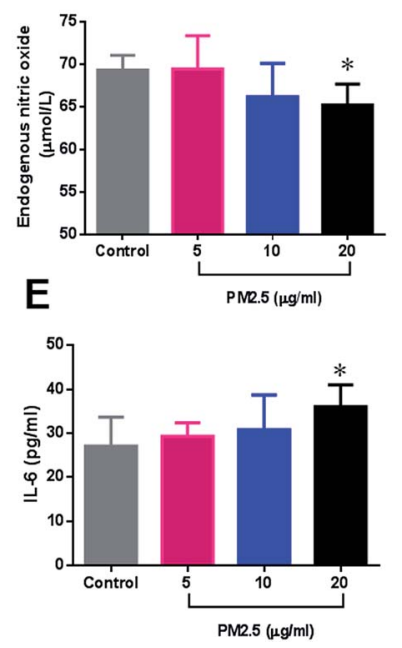

B
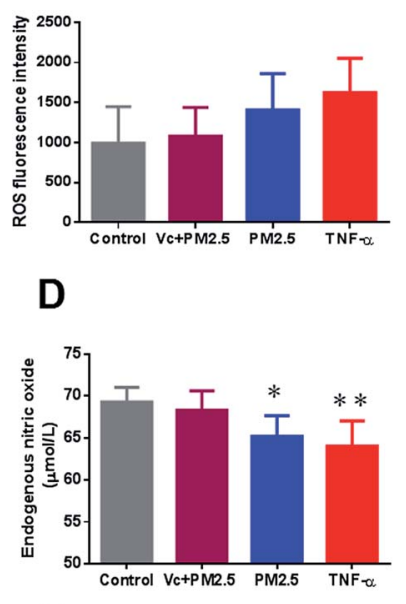

$\mathbf{F}$

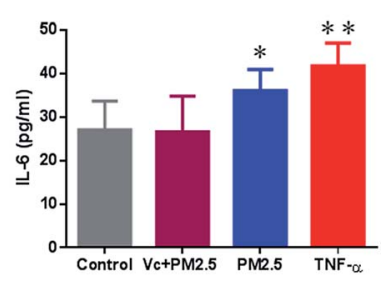

Fig. 7 FPM stimulates endothelial inflammation based on a 3D human microvascular model: FPMs $\left(5,10\right.$ and $20 \mu \mathrm{g} \mathrm{ml}^{-1}$ ) stimulate intravascular ROS (A), endothelial NO (C) and IL-6 (E); stimulation of intravascular ROS (B), endothelial NO (D) and IL-6 (F) by FPM/VC (vitamin C-0.1 mg ml$\left.{ }^{-1}, F P M-20 \mu \mathrm{g} \mathrm{ml}^{-1}\right)$, FPM $\left(20 \mu \mathrm{g} \mathrm{ml}^{-1}\right)$ and TNF$\alpha\left(2 \mathrm{ng} \mathrm{ml}^{-1}\right)(* P<0.05, * * P<0.01$, compared with the control).

decreased following FPM exposure (Fig. 7C). And FPM (20 $\mu \mathrm{g}$ $\left.\mathrm{ml}^{-1}\right)$ significantly affected the normal expression of NO $(p<$ 0.05) as compared to the control group (Fig. 7C). However, an antioxidant (vitamin C) effectively suppressed the decrease in NO resulting from FPM stimulation. All these results indicate that FPM may stimulate intravascular oxidative stress, which further disrupts the normal expression of endothelial NO. Endothelial NO is a strong inhibitor of platelet adhesion, activation and aggregation. ${ }^{66}$ A deficiency of bioactive NO contributes to platelet over-activation which is associated with thrombosis. ${ }^{67}$

Tumor Necrosis Factor- $\alpha$ (TNF- $\alpha)$ is a major proinflammatory cytokine involved in early inflammatory events. ${ }^{68}$ Based on a 3D-human microvascular model, we saw that TNF- $\alpha$ triggered a series of inflammatory responses, which included the upregulation of ROS, endothelial NO and IL-6 (Fig. 7B, D and F). These results indicated that the proinflammatory cytokine plays a major role in human endothelial dysfunction. In this study, we investigated the procoagulant mechanisms and assumed that particulate matter might accelerate inflammation-induced thrombosis, since we found that a 3D-microvascular network treated with a dose of FPM exhibited an increase in IL-6 levels (Fig. 7E). FPM $\left(20 \mu \mathrm{g} \mathrm{ml}{ }^{-1}\right)$ significantly increased IL-6 expression $(p<0.05)$ as compared to

the control group (Fig. 7E). And an antioxidant (vitamin C) effectively suppressed the increase in IL-6 resulting from FPM stimulation (Fig. 7F). IL-6 is a cytokine expressed in response to a wide variety of inflammatory stimuli, and is capable of activating the coagulation system and down-regulating important physiological anticoagulant pathways. ${ }^{69}$ Our results suggest that FPM are expected to enhance endothelial inflammation which might in turn accelerate coagulation that is associated with thrombosis.

In the past decade, researchers have already developed a series of epidemiologic evidence showing correlation with cardiovascular and particulate air pollution. Based on animal model, research further indicated that oxidant stress induced endothelial dysfunction plays an important role in pollution particle involved cardiovascular diseases. However, animal results are unable to fully embody human's response to pollution particles. A human organotypic 3D microvessel model is an attractive alternative to traditional animal models. Based on microfluidic chip, this model is always used to study vascular diseases. The advantage is that the pathogenesis can be observed visually in vitro platform. In this study, we are first to use this model to evaluate vascular toxicity of ambient fine particles. Our results suggest that FPM are capable form intravascular accumulation, they are expected to enhance endothelial inflammation which might in turn accelerate coagulation that is associated with thrombosis. It reveals one possible way to explain particulate matter involving cardiovascular disease.

\section{Methods}

\subsection{FPM collection and characterization}

The airborne particles were collected on quartz filters using a high-volume PM2.5 sampler (Anderson, USA). The sampling site was located in Wuhan City, the largest industrial city in Central China. Each quartz filter was cut into smaller pieces of about $2 \mathrm{~cm}^{2}$. These pieces were put into a sterilized beaker with $90 \mathrm{ml}$ sterilized pure water. A $20 \mathrm{~min}$ sonification was carried out below $20{ }^{\circ} \mathrm{C}$. Samples were then filtered using a sterilized filter $(<1 \mu \mathrm{m})$ to remove larger particles as well as any fibers from the quartz. Filtration could remove most part of Si element which may come from quartz fibers. It could avoid any quartz fibers attached on ambient fine particulate matters. So as to ensure the detected $\mathrm{Si}$ elements are very likely just from ambient fine particulate matters. The collected FPM suspensions were freeze dried in a vacuum for $24 \mathrm{~h}$ and stored at $-20{ }^{\circ} \mathrm{C}$. The morphology and size of FPMs were observed using atomic force microscopy (SPM 3100, Veeco Instruments, Inc., USA). Elements of FPM were analyzed by using SEM-EDS (energy dispersive spectroscopy) analysis.

\subsection{Cell culture and solution preparation of fibrinogen and thrombin}

GFP-HUVECs (Neuromics, USA) or non GFP-HUVECs (Lonza, USA) were cultured up to passage 6 in endothelial growth medium (EGM-2, Lonza). HLFs were cultured up to passage 10 in fibroblast growth medium (FGM-2, Lonza). Cell cultures were 
grown to $80 \%$ confluence prior to passage and use in experiments. DPBS (Gibco, USA) was preheated at $37^{\circ} \mathrm{C}$ for $2 \mathrm{~h}$. The fibrinogen solution (15 $\mathrm{mg} \mathrm{ml}^{-1}$ ) was prepared by dissolving $15 \mathrm{mg}$ bovine fibrinogen (Sigma, USA) in the preheated DPBS. Thrombin (100 U, Sigma, USA) solution was dissolved in $1 \mathrm{ml}$ DPBS which contained $0.1 \%$ BSA.

\subsection{Microvascular network formation}

PBS, Trypsin-EDTA and endothelial cell growth medium were preheated in the $37{ }^{\circ} \mathrm{C}$ water bath. The media was removed and discarded from the cell flask and the cells then rinsed with PBS. $1 \mathrm{ml}$ of Trypsin-EDTA was added to the flask, mixed briefly, and incubated at $37{ }^{\circ} \mathrm{C}$ for a few minutes to release the cells. HUVECs were resuspended at a concentration of 12 million cells per ml. HLFs were resuspended at a concentration of 6 million cells per ml. $1 \mu$ thrombin solution $\left(100 \mathrm{U} \mathrm{ml}^{-1}\right)$ was mixed with $20 \mu$ l fibrinogen solution (15 $\mathrm{mg} \mathrm{ml}^{-1}$, Sigma, USA), $79 \mu \mathrm{l}$ HUVECs $\left(12 \times 10^{6}\right.$ cell per ml $)$ and $20 \mu \mathrm{l}$ HLFs $\left(6 \times 10^{6}\right.$ cell per $\mathrm{ml}$ ). $10 \mu \mathrm{l}$ of this mixture was immediately injected into the central gel channel of the microfluidic device. Devices were placed in a humidified incubator at $37^{\circ} \mathrm{C}$, and the cell mixture was allowed to gel for 40 minutes. The EGM-2 media were then loading in the media channels, and the media were replaced every $24 \mathrm{~h}$. The microfluidic device was cultured in a humidified incubator for 4 days at $37{ }^{\circ} \mathrm{C}$ and $5 \% \mathrm{CO}_{2}$. During the progress of microvascular development, images of the 3D HUVEC networks were captured by a camera linked to a confocal microscope (Olympus IX81, USA). Images of the fluorescent micro-vessels were captured by a confocal microscope (Olympus IX81, USA).

\subsection{Perfusion measurement of microvasular networks}

Perfusion of 3D cultured microvessels is almost exclusively the domain of microfluidic techniques. In this research, perfusion capability has been measured for the microvascular networks in the microfluidic device. Perfusion of the microvasculature was measured via fluorescent microparticle (Invitrogen, USA) flow through the microvascular network consisting of continuous and perfusable lumens. Briefly, the fluorescent microparticle solution was diluted 100-fold with EGM-2 medium. Fill $20 \mu \mathrm{l}$ of this medium into any two syringe barrels that are connected to the same media channel. Fluid flow in a media channel creates a pressure drop along the channel. The movement of the fluorescent microparticles within the generated perfusable lumens was captured using a confocal microscope (Olympus IX81, USA). Microparticle movement was monitored by a microfluid device from the upstream, downstream and panorama views.

\subsection{Measurement of FPM associated oxidative stress and inflammation responses}

FPM was dissolved in the EGM-2 medium with concentrations of 5, 10 and $20 \mu \mathrm{g} \mathrm{ml} \mathrm{m}^{-1}$. Exposure solutions of tumor necrosis factor- $\alpha$ (TNF- $\alpha$ ) (Sigma, USA) and FPM-vitamin C were also prepared. The final exposure concentrations were $2 \mathrm{ng} \mathrm{ml}$ (TNF- $\alpha$ ), $0.1 \mathrm{mg} \mathrm{ml} \mathrm{m}^{-1}$ (vitamin C) and $20 \mu \mathrm{gl}^{-1}$ (FPM), respectively. Each kind of exposure solution was loaded into the microvascular networks for 24 hours at $37{ }^{\circ} \mathrm{C}$ and $5 \% \mathrm{CO}_{2}$. The measurement of ROS, endothelial NO and IL- 6 were performed as described here. Devices were washed three times with the EGM-2 medium, and the washing medium collected for assessment of endothelial NO and IL-6. The concentrations of endothelial NO and IL-6 were measured using ELISA kits according to the manufacturer's instructions (Nanjing Jiancheng, China). ROS were measured using oxidation-sensitive fluorescent DCFH-DA (Merck KGaA, Germany), which is a non-fluorescent compound that is freely taken up by cells and hydrolyzed by esterases to $2^{\prime}, 7^{\prime}$-dichlorodihydrofluorescein (DCFH). Non GFP-HUVECS was co-cultured with HLFs, fibrinogen and thrombin, and the mixtures then loaded into microfluidic devices. The formed microvasculature was used in ROS assessment. Devices were washed once with PBS, and the fluorescent agent was loaded into the microvascular networks for $20 \mathrm{~min}$ at $37{ }^{\circ} \mathrm{C}$ and $5 \% \mathrm{CO}_{2}$. Devices were washed twice with PBS, and loading EGM-2 medium. The fluorescent images were captured by confocal microscope (Olympus IX81, USA). The fluorescence intensity of ROS expression was analyzed using ImageJ software.

\subsection{Statistical analysis}

GraphPad Prism software was used for statistical analysis of the experimental data, and for graphing the results. All data are presented as the mean \& standard deviation (S.D.). The presence of statistical differences between groups was determined by ANOVA. The method of least significant difference (LSD) was used to compare the effects between each exposure group and the control. $p<0.05$ and $p<0.01$ were considered significant.

\section{Conclusions}

In summary, we developed a 3D functional human microvascular network in a microfluidic device. The microvessels effectively mimic the characteristic morphological and partly simulate some physiological functions of human blood vessels. The perfusable microvasculature allows for the delivery of nutrients, oxygen, as well as flow-induced mechanical stimuli into the luminal space of the endothelium. Following with fluid flow, FPM was exposed to microvessel by 3D culture of HUVEC. Our results demonstrated that intravascular accumulation of FPM could enhance ROS generation, disrupt NO expression and activate endothelial inflammation. However, administering an antioxidant effectively suppressed any FPM induced intravascular inflammation. Based on the obtained data, we concluded that FPM induced oxidative stress might generate NO deficiency and accelerate intravascular inflammation. All of these are considered to accelerate coagulation which is associated with thrombosis. A human organotypic 3D microvessel model is an attractive alternative to traditional animal models. It could partly simulate the physiological responses of human vessels to FPM stimulation. 3D organotypic cell culture provides a bridge for how research outcomes can map to humans. This simple and versatile platform provides for a wide range of applications 
for vascular physiology studies of PM involving cardiovascular disease.

\section{Conflicts of interest}

The authors declare that they have no competing interests.

\section{Acknowledgements}

This work was supported by National Natural Science Foundation of China (No. 21607117).

\section{Notes and references}

1 F. J. Kelly and J. C. Fussell, Environ. Geochem. Health, 2015, 37, 631-649.

2 Y. Du, X. Xu, M. Chu, Y. Guo and J. Wang, J. Thorac. Dis., 2016, 8, E8-E19.

3 D. W. Dockery, C. A. Pope, X. Xu, J. D. Spengler, J. H. Ware, M. E. Fay, B. G. Ferris and F. E. Speizer, N. Engl. J. Med., 1993, 329, 1753-1759.

4 C. A. Pope, R. T. Burnett, M. J. Thun, E. E. Calle, D. Krewski, K. Ito and G. D. Thurston, J. Am. Med. Assoc., 2002, 287, 11321141.

5 C. Liu, J. Cai, L. Qiao, H. Wang, W. Xu, H. Li, Z. Zhao, R. Chen and H. Kan, Environ. Sci. Technol., 2017, 51, 8128-8137.

6 A. Viehmann, S. Hertel, K. Fuks, L. Eisele, S. Moebus, S. Möhlenkamp, M. Nonnemacher, H. Jakobs, R. Erbel, K. H. Jöckel, B. Hoffmann and N. Heinz, Occup. Environ. Med., 2015, 72, 656-663.

7 N. R. Yacobi, F. Fazllolahi, Y. H. Kim, A. Sipos, Z. Borok, K. J. Kim and E. D. Crandall, Air Qual., Atmos. Health, 2011, 4, 65-78.

8 R. W. Baldauf, R. B. Devlin, P. Gehr, R. Giannelli, B. H. Sipple, H. Jung, G. Martini, J. McDonald, J. D. Sacks and K. Walker, Int. J. Environ. Res. Public Health, 2016, 13, 1054.

9 N. R. Labiris and M. B. Dolovich, Br. J. Clin. Pharmacol., 2003, 56, 588-599.

10 L. K. Braydich-Stolle, E. K. Breitner, K. K. Comfort, J. J. Schlager and S. M. Hussain, Langmuir, 2014, 30, 15309-15316.

11 P. D. Pino, B. Pelaz, Q. Zhang, P. Maffre, G. U. Nienhausbc and W. J. Parak, Mater. Horiz., 2014, 1, 301-313.

12 E. H. Pilkington, Y. Xing, B. Wang, A. Kakinen, M. Wang, T. P. Davis, F. Ding and P. C. Ke, Sci. Rep., 2017, 7, 2455.

13 Y. K. Lee, E. J. Choi, S. H. Kim and D. W. Khang, Int. J. Nanomed., 2015, 10, 97-113.

14 A. Gojova, B. Guo, R. S. Kota, J. C. Rutledge, I. M. Kennedy and A. I. Barakat, Environ. Health Perspect., 2007, 115, 403409.

15 M. Ratoi, P. H. M. Hoetb, A. Crossleyc and P. Dobson, $R S C$ Adv., 2014, 4, 20573-20581.

16 J. Zhao, Y. Xie, R. Jiang, K. Han and W. Song, Hum. Exp. Toxicol., 2011, 30, 1828-1839.

17 J. Dai, C. Sun, Z. Yao, W. Chen, L. Yu and M. Long, FEBS Open Bio, 2016, 6, 720-728.
18 L. Badimon and G. Vilah, J. Intern. Med., 2014, 276, 618-632. 19 S. S. Song, B. Y. Xia, J. Chena, J. Yangd, X. Shena, S. J. Fana, M. 1. Guo, Y. M. Sun and X. D. Zhang, $R S C A d v$., 2014, 4, 42598-42603.

20 N. Shanks, R. Greek and J. Greek, Philosophy, Ethics, and Humanities in Medicine, 2009, 4, 1-20.

21 D. Antoni, H. Burckel, E. Josset and G. Noel, Int. J. Mol. Sci., 2015, 16, 5517-5527.

22 D. Huh, J. A. Hamilton and D. E. Ingber, Trends Cell Biol., 2011, 21, 745-754.

23 S. Kim, H. Lee, M. Chung and N. L. Jeon, Lab Chip, 2013, 13, 1489-1500.

24 M. B. Chena, J. A. Whislera, J. S. Jeona and R. D. Kamm, Integr. Biol., 2013, 5, 1262-1271.

25 X. Meng, P. Leslie, Y. Zhang and J. Dong, SpringerPlus, 2014, 3,80 .

26 A. Mata, E. J. Kim, C. A. Boehm, A. J. Fleischman, G. F. Muschler and S. Roy, Biomaterials, 2009, 30, 4610-4617.

27 Y. Haraguchi, T. Shimizu, M. Yamato and T. Okano, RSC Adv., 2012, 2, 2184-2190.

28 Q. Smith and S. Gerecht, Curr. Opin. Chem. Eng., 2014, 3, 4250.

29 N. Gupta, J. R. Liu, B. Patel, D. E. Solomon, B. Vaidya and V. Gupt, Bioeng. Transl. Med., 2016, 1, 63-81.

30 X. Wang, Z. Liu and Y. Pang, RSC Adv., 2017, 7, 29966-29984. 31 C. M. Sakolish and G. J. Mahler, RSC Adv., 2017, 7, 42164225.

32 G. S. Jeong, S. Han, Y. Shin, G. H. Kwon, R. D. Kamm, S. H. Lee and S. Chung, Anal. Chem., 2011, 83, 8454-8459.

33 Y. Shi, Y. Ji, H. Sun, F. Hui, J. Hu, Y. Wu, J. Fang, H. Lin, J. Wang, H. Duan and M. Lanza, Sci. Rep., 2015, 5, 11232.

34 H. M. Braakhuis, M. Park, I. Gosens, W. H. De Jong and F. R. Cassee, Part. Fibre Toxicol., 2014, 11, 18.

35 A. N. Ilinskaya and M. A. Dobrovolskaia, Nanomedicine, 2013, 8, 969-981.

36 A. Akhtar, Cambridge Quarterly of Healthcare Ethics, 2015, 24, 407-419.

37 I. W. Y. Mak, N. Evaniew and M. Ghert, Am. J. Transl. Res., 2014, 6, 114-118.

38 S. Breslin and L. O'Driscoll, OncoTargets Ther., 2016, 7, 45745-45756.

39 S. Bersini and M. Moretti, J. Mater. Sci.: Mater. Med., 2015, 26, 5520-5525.

40 X. Y. Wang, Z. H. Jin, B. W. Gan, S. W. Lv, M. Xie and W. H. Huang, Lab Chip, 2014, 14, 2709-2716.

41 Y. Li, H. Meng, Y. Liu and B. P. Lee, Sci. World J., 2015, 2015, 685690.

42 C. H. Liu, S. C. Niranjan, J. W. Clark Jr, K. Y. San, J. B. Zwischenberger and A. Bidani, J. Appl. Physiol., 1985, 84, 1447-1469.

43 P. Vaupel, F. Kallinowski and P. Okunieff, Cancer Res., 1989, 49, 6449-6465.

44 R. L. Twardowski and L. D. Black, Ann. Biomed. Eng., 2014, 42, 1074-1084.

45 J. S. Esser, S. Rahner, M. Deckler, C. Bode, C. Patterson and M. Moser, Arterioscler., Thromb., Vasc. Biol., 2015, 35, 358-367. 
46 A. C. Newman, M. N. Nakatsu, W. Chou, P. D. Gershon and C. C. W. Hughes, Mol. Biol. Cell, 2011, 22, 3791-3800.

47 I. Choi, S. Lee and Y. K. Hong, Cold Spring Harbor Perspect. Med., 2012, 2, a006445.

48 G. Ackland, M. P. W. Grocott and M. G. Mythen, Crit. Care, 2000, 4, 269-281.

49 J. M. Sosa, N. D. Nielsen, S. M. Vignes, T. G. Chen and S. S. Shevkoplyas, Clin. Hemorheol. Microcirc., 2014, 57, 291-305.

50 L. Kim, Y. C. Toh, J. Voldman and H. Yu, Lab Chip, 2007, 7, 681-694.

51 V. van Duinen, S. J. Trietsch, J. Joore, P. Vulto and T. Hankemeier, Curr. Opin. Biotechnol., 2015, 35, 118-126.

52 M. P. Leal, C. Muñoz-Hernández, C. C. Berryb and M. L. García-Martín, RSC Adv., 2015, 5, 76883-76889.

53 S. W. Jones, R. A. Roberts, G. R. Robbins, J. L. Perry, M. P. Kai, K. Chen, T. Bo, M. E. Napier, J. P. Y. Ting, J. M. DeSimone and J. E. Bear, J. Clin. Invest., 2013, 123, 3061-3073.

54 I. B. Magaña, R. B. Yendluri, P. Adhikari, G. P. Goodrich, J. A. Schwartz, E. A. Sherer and D. P. O'Neal, Ther. Delivery, 2015, 6, 777-783.

55 R. A. D. Arancon, S. H. T. Lin, G. Chen, C. S. K. Lin, J. Lai, G. Xud and R. Luque, RSC Adv., 2014, 4, 17114-17119.

56 D. Lu and G. S. Kassab, J. R. Soc., Interface, 2011, 8, 1379-1385. 57 L. Badimon, T. Padró and G. Vilahur, European Heart Journal: Acute Cardiovascular Care, 2012, 1, 60-74.
58 C. T. Esmon, J. Thromb. Haemostasis, 2003, 1, 1343-1348.

59 J. Johne, C. Blume, P. M. Benz, M. Pozgajová, M. Ullrich, K. Schuh, B. Nieswandt, U. Walter and T. Renné, Biol. Chem., 2006, 387, 173-178.

60 K. Aksu, A. Donmez and G. Keser, Curr. Pharm. Des., 2012, 18, 1478-1493.

61 Y. Ma, Z. Ma, S. Yin, X. Yan and J. Wang, Chemosphere, 2017, 167, 454-461.

62 H. Lum and K. A. Roebuck, Am. J. Physiol.: Cell Physiol., 2001, 280, C719-C741.

63 M. Mittal, M. R. Siddiqui, K. Tran, S. P. Reddy and A. B. Malik, Antioxid. Redox Signaling, 2014, 20, 1126-1167.

64 P. Rajendran, T. Rengarajan, J. Thangavel, Y. Nishigaki, D. Sakthisekaran, G. Sethi and I. Nishigaki, Int. J. Biol. Sci., 2013, 9, 1057-1069.

65 H. Ding and C. R. Triggle, Vasc. Health Risk Manage., 2005, 1, 55-71.

66 S. P. Watson, Curr. Pharm. Des., 2009, 15, 1358-1372.

67 J. W. Yau, H. Teoh and S. Verma, BMC Cardiovasc. Disord., $2015,15,130$.

68 X. Xu, D. Huang, W. Liu, Z. Sheng, K. Liang, D. Li, D. Zhao, Y. Ma, K. Zhang, T. Hayat, N. S. Alharbif and W. Li, RSC Adv., 2017, 7, 34699-34704.

69 M. Levi, T. van der Poll and M. Schultz, Semin. Immunopathol., 2012, 34, 167-179. 